\title{
SUMMABILITY METHODS FOR INDEPENDENT, IDENTICALLY DISTRIBUTED RANDOM VARIABLES 1
}

\author{
TZE LEUNG LAI
}

ABSTRACT. In this paper, we present certain theorems concerning the Cesaro $(C, \alpha)$, Abel $(A)$, Euler $(E, q)$ and Borel $(B)$ summability of $\Sigma Y$, where $Y_{i}=X_{i}-X_{i-1}, X_{0}=0$ and $X_{1}, X_{2}, \ldots$ are i.i.d. random variables. While the Kolmogorov strong law of large numbers and the Hartman-Wintner law of the iterated logarithm are related to $(C, 1)$ summability and involve the finiteness of, respectively, the first and secord moments of $X_{1}$, their analogues for Euler and Borel summability involve different moment conditions, and the analogues for $(C, \alpha)$ and Abel summability remain essentially the same.

Let $X_{1}, X_{2}, \ldots$ be independent, identically distributed (i.i.d.) random variables. Let $Y_{n}=X_{n}-X_{n-1}\left(X_{0}=Y_{0}=0\right)$. Kolmogorov's strong law of large numbers asserts that $E X_{1}=\mu$ iff $\Sigma Y_{i}$ is a.e. $(C, 1)$ summable to $\mu$, i.e., the $(C, 1)$ limit of $X_{n}$ is $\mu$ a.e. By the well-known inclusion theorems involving Cesaro and Abel summability (cf. [5, Theorems 43 and 55]), this implies that $\Sigma Y_{i}$ is a.e. $(C, \alpha)$ summable to $\mu$ for any $\alpha \geq 1$ and that $\Sigma Y_{i}$ is a.e. (A) summable to $\mu$. In fact, the converse also holds in the present case, and we have the following theorem.

Theorem 1. If $X_{1}, X_{2}, \cdots$ is a sequence of i.i.d. random variables and $\alpha \geq 1$ and $\mu$ are given real numbers, then the following statements are equiv. alent:

(1) $E X_{1}=\mu$.

(2) $X_{n} \rightarrow \mu(C, 1)$ a.e., i.e., $\lim _{n \rightarrow \infty}(1 / n)\left(X_{1}+\cdots+X_{n}\right)=\mu$ a.e.

(3) $X_{n} \rightarrow \mu(C, \alpha)$ a.e., i.e., $\lim _{n \rightarrow \infty} \sum_{i=1}^{n-1}\left(\begin{array}{c}i+a-1 \\ i\end{array}\right) X_{n-i} /\left(\begin{array}{c}n+\alpha \\ n\end{array}\right)=\mu$ a.e., where $\left(\begin{array}{c}j+\beta \\ j\end{array}\right)=(\beta+1) \cdots(\beta+j) /(j !)$.

(4) $X_{n} \rightarrow \mu(A)$ a.e., i.e., $\lim _{\lambda \rightarrow 1-}(1-\lambda) \sum_{i=1}^{\infty} \lambda^{i} X_{i}=\mu$ a.e.

Received by the editors June 5, 1973 and, in revised form, November 12, 1973.

AMS (MOS) subject classifications (1970). Primary 60F15; Secondary 40D25, $40 \mathrm{G} 10$.

Key words and phrases. Summability, strong law of large numbers, law of the iterated logarithm.

1 Research supported by PHS Grant GM-16895-03. 
Proof. The implications (2) $\Rightarrow(3) \Rightarrow(4)$ are well known (cf. [5, pp. 96, $100,108])$. We shall now prove that $(4) \Rightarrow(1)$. By $(4)$,

$$
\lim _{m \rightarrow \infty} \frac{1}{m} \sum_{n=1}^{\infty} e^{-n / m} X_{n}^{s}=0 \text { a.e., }
$$

where $X_{n}^{s}=X_{n}-X_{n}^{\prime}$ with $X_{n}^{\prime}, n \geq 1$, and $X_{n}, n \geq 1$, being i.i.d. Let

$$
Y_{m}=\frac{1}{m} \sum_{n=1}^{m} e^{-n / m} X_{n}^{s}, \quad Z_{m}=\frac{1}{m} \sum_{n=m+1}^{\infty} e^{-n / m} X_{n}^{s} .
$$

Then $Y_{m}+Z_{m} \stackrel{P}{\longrightarrow} 0, Y_{m}$ and $Z_{m}$ are independent and symmetric. Therefore it follows easily from the Lévy inequality [8, p. 247] that $Z_{m} \stackrel{P}{\longrightarrow} 0$. Since $Z_{m}$ and $\left(Y_{1}, \ldots, Y_{m}\right)$ are independent and $Y_{m}+Z_{m} \rightarrow 0$ a.e., $Z_{m} \stackrel{P}{\longrightarrow} 0$, we obtain by Lemma 3 of [2] that $Y_{m} \rightarrow 0$ a.e. Letting $Y_{m}^{(1)}=$ $Y_{m}^{m}-(\mathrm{em})^{-1} X_{m}^{s}$, since $(\mathrm{em})^{-1} X_{m}^{s} \stackrel{P}{\longrightarrow} 0$, we again obtain by Lemma 3 of [2] that $X_{m}^{s} / m \rightarrow 0$ a.e. By the Borel-Cantelli lemma, this implies that $E\left|X_{1}\right|$ $<\infty$. As established before, we then have $X_{n} \rightarrow E X_{1}(A)$ and so by (4), $\mu=E X_{1}$.

The classical law of the iterated logarithm gives us the rate at which the convergence in (2) takes place. Gaposhkin [4] has established the law of the iterated logarithm for the $(C, \alpha)$ and Abel methods in the case where $X_{1}$ is bounded. Actually by using either the Hartman-Wintner truncation scheme [6] or Strassen's strong invariance principle [9], we can extend Gaposhkin's results to the case where $0<E X_{1}^{2}<\infty$. The extension is the sharpest possible in the sense that its converse also holds, and this converse can be established by a modification of Feller's argument in [3]. The details of the proof are omitted here, and we simply state the results in the follow ing theorem.

Theorem 2. With the same notation as in Theorem 1, for any given positive number $\sigma$, the following statements are equivalent:

(5) $E X_{1}=0, E X_{1}^{2}=\sigma^{2}$.

(6) $\lim \sup _{n \rightarrow \infty}\left|\Sigma_{1}^{n} X_{i}\right| /\{2 n \log \log n\}^{1 / 2}=\sigma a \cdot e$.

(7) $\lim \sup _{n \rightarrow \infty}\left|\sum_{i=1}^{n}\left(\begin{array}{c}i+a-1 \\ i\end{array}\right) X_{n-i}\right| /\left\{2 n^{2 a-1} \log \log n\right\}^{1 / 2}=\sigma /\left\{(2 \alpha-1)^{1 / 2} \Gamma(\alpha)\right\}$ a.e.

(8) $\lim \sup _{\lambda \rightarrow 1-}(1-\lambda)^{1 / 2}\left|\sum_{i=1}^{\infty} \lambda^{i} X_{i}\right| /\{\log |\log (1-\lambda)|\}^{1 / 2}=$ o a.e.

Our primary interest in this paper lies in the analogue of the law of the iterated logarithm for the Euler and Borel methods of summation (cf. [5, 
Chapters 8 and 9]). Chow [1] has shown that unlike the Cesaro and Abel methods which require $E\left|X_{1}\right|<\infty$ for summability, the Euler and Borel methods require $E X_{1}^{2}<\infty$ for summability. Specifically, if $X_{1}, X_{2}, \cdots$ are i.i.d., then the following statements are equivalent:

(9) $E X_{1}=\mu, E X_{1}^{2}<\infty$.

(10) $X_{n} \rightarrow \mu(E, q)$ for some (or equivalently for every) $q>0$, i.e.,

$$
\lim _{n \rightarrow \infty}(q+1)^{-n} \sum_{i=1}^{n}\left(\begin{array}{l}
n \\
i
\end{array}\right) q^{n-i} X_{i}=\mu \text { a.e. }
$$

(11) $X_{n} \rightarrow \mu(B)$, i.e.,

$$
\lim _{\lambda \rightarrow \infty} e^{-\lambda} \sum_{i=1}^{\infty} \frac{\lambda^{i}}{i !} X_{i}=\mu \quad \text { a.e. }
$$

The following theorem gives the $r$ ate at which the convergence in (10) or (11) takes place. It is interesting to compare the result with Theorem 2 .

Theorem 3. Suppose $q>0, \sigma>0$ and $X_{1}, X_{2}, \ldots$ are i.i.d. random variables. Then the following statements are equivalent:

(12) $E X_{1}=0, E X_{1}^{2}=\sigma^{2}, E X_{1}^{4}\left(\log ^{+}\left|X_{1}\right|+1\right)^{-2}<\infty$.

(13) $\lim \sup _{\lambda \rightarrow \infty}(\pi \lambda)^{1 / 4}(\log \lambda)^{-1 / 2}\left|\sum_{i=1}^{\infty} e^{-\lambda}\left(\lambda^{i} / i !\right) X_{i}\right|=\sigma / \sqrt{ } 2$ a.e.

(14) $\lim \sup _{n \rightarrow \infty}(\pi q n)^{1 / 4}(\log n)^{-1 / 2}\left|\sum_{i=1}^{n}(q+1)^{-n}\left(\begin{array}{c}n \\ i\end{array}\right) q^{n-i} X_{i}\right|$ $=\sigma(1 / 2(q+1))^{1 / 2}$ a.e.

Lemma. Let $X_{1}, X_{2}, \cdots$ be independent random variables with $E X_{n}=$ $0, E X_{n}^{2}=\sigma_{n}^{2}$ and $\lim _{n \rightarrow \infty} \sigma_{n}^{2}=\sigma^{2}>0$. Suppose for $j \geq j_{0}$, there exists $r_{j} \geq$ 0 such that $r_{j}=o\left(j^{1 / 4}(\log j)^{-1 / 2}\right)$ and

$$
\exp \left\{t^{2} \sigma_{j}^{2}\left(1-|t| r_{j}\right) / 2\right\} \leq E \exp \left(t X_{j}\right) \leq \exp \left\{t^{2} \sigma_{j}^{2}\left(1+|t| r_{j} / 2\right) / 2\right\}
$$

whenever $|t| r_{j} \leq 1$. Then for any $c>0$ and $\alpha>1 / 2$,

(16) $\quad \lim _{M \rightarrow \infty} \sup _{M \rightarrow}\left(\frac{\pi M}{2 c}\right)^{-1 / 4}(\log M)^{-1 / 2} \sum_{|m-M| \leq M^{\alpha}} X_{m} \exp \left(\frac{-c(m-M)^{2}}{M}\right)=\sigma$ a.e.

Proof. To simplify the notation, we shall set $h=m-M$. Let $0<\delta<$ $\delta^{\prime}<1$ such that $\left(1-\delta^{\prime}\right)^{2}(1+\delta)<1$. Choose $\zeta>1$ such that

$$
\delta>3\left(2 \int_{\zeta}^{\infty} e^{-2 c t^{2}} d t\right)^{1 / 2} \text { and } \int_{-\zeta}^{\zeta} e^{-2 c t^{2}} d t>(1-\delta)^{2}(\pi / 2 c)^{1 / 2}
$$




$$
\begin{aligned}
& s_{M}^{2}=\sum_{\zeta \sqrt{ } M<|h| \leq M^{\alpha}}\left(\exp \left(-2 c h^{2} / M\right)\right) \sigma_{m}^{2} \sim 2 \sigma^{2} \sqrt{ } M \int_{\zeta}^{\infty} \exp \left(-2 c t^{2}\right) d t, \\
& Y_{M}=\left(\frac{1}{s_{M}}\right)_{\zeta \bigvee M<|h| \leq M^{\alpha}} \sum_{\zeta}\left(\exp \left(-c h^{2} / M\right)\right) X_{m} .
\end{aligned}
$$

Using (15) and Kolmogorov's (upper) exponential bounds (cf. [10, Lemma 1]), we obtain that for all large $M$,

$$
\begin{aligned}
P\left[\sum_{\zeta \backslash M<|h| \leq M}\left(\exp \left(-c h^{2} / M\right)\right) X_{m} \geq \delta \sigma M^{1 / 4}(\log M)^{1 / 2}\right] \\
\leq P\left[Y_{M} \geq 3(\log M)^{1 / 2}\right] \leq \exp \{-(9 / 4) \log M\} .
\end{aligned}
$$

Replacing $X_{m}$ by $-X_{m}$ in (17) gives a similar inequality, and an application of the Borel-Cantelli lemma gives

$$
\limsup _{M \rightarrow \infty} M^{-1 / 4}(\log M)^{-1 / 2}\left|\sum_{\zeta \sqrt{ } M<|h| \leq M^{\alpha}}\left(\exp \left(-c h^{2} / M\right)\right) X_{m}\right| \leq \delta \sigma \quad \text { a.e. }
$$

Choose $\eta>\zeta^{2}$ and set $m_{k}=\left[\eta k^{2}\right]$. Using (15) and Kolmogorov's (lower) exponential bounds (cf. [10, Lemma 1]), it can be shown that for all large $k$,

$$
P\left[\left(\frac{\pi m_{k}}{2 c}\right)^{-1 / 4} \sum_{|h| \leq \zeta \sqrt{ } m_{k}} X_{m} \exp \left(\frac{-c h^{2}}{m_{k}}\right) \geq(1-\delta) \sigma(2 \log k)^{1 / 2}\right]
$$

$$
\geq \exp \left\{-(1+\delta)\left(1-\delta^{\prime}\right)^{2} \log k\right\}
$$

Now the $\sigma$-fields $\mathcal{F}_{k}=\mathscr{B}\left(X_{m}:\left|m-m_{k}\right| \leq \zeta \vee m_{k}\right)$ are independent for all large $k$. Hence it follows from (19) and the Borel-Cantelli lemma that

$$
\limsup _{k \rightarrow \infty}\left(\frac{\pi m_{k}}{2 c}\right)^{-1 / 4}(2 \log k)^{-1 / 2} \sum_{|h| \leq \zeta \sqrt{ } m_{k}} X_{m} \exp \left(\frac{-c h^{2}}{m_{k}}\right) \geq(1-\delta) \sigma \text { a.e. }
$$

Since $2 \log k \sim \log m_{k}$ and $\delta$ is arbitrary, we obtain from (18) and (20) that the lim sup in (16) is $\geq \sigma$ a.e.

To prove that the lim sup in $(16)$ is $\leq \sigma$ a.e., let $\eta_{k}=(\log k)^{-2}, M_{k}=$ $\left[\eta_{k} k^{2}\right]$. An application of Kolmogorov's (upper) exponential bounds gives 
(21) $\sum P\left[\left(\frac{\pi M_{k}}{2 c}\right)^{-1 / 4} \sum_{|h| \leq \zeta \sqrt{ } M_{k}} X_{m} \exp \left(\frac{-c h^{2}}{M_{k}}\right) \geq(1+\delta) \sigma(2 \log k)^{1 / 2}\right]<\infty$.

Obviously (21) still holds with $X_{m}$ replaced by $-X_{m}$, and noting that $\log M_{k} \sim 2 \log k$, we obtain that

$\left.\lim _{k \rightarrow \infty} \sup _{\left(\frac{\pi M_{k}}{2 c}\right.}\right)^{-1 / 4}\left(\log M_{k}\right)^{-1 / 2}\left|\sum_{|h| \leq \zeta \sqrt{ } M_{k}} X_{m} \exp \left(\frac{-c b^{2}}{M_{k}}\right)\right| \leq(1+\delta) \sigma$ a.e.

We now assert that

$$
\lim _{k \rightarrow \infty} \sup _{k} M_{k}^{-1 / 4}\left(\log M_{k}\right)^{-1 / 2} \max _{M_{k}<M \leq M_{k+1}}\left|U_{k, M}\right| \leq 6 \delta \text { a.e., }
$$

where we define, for $M_{k}<M \leq M_{k+1}$,

$$
\begin{aligned}
& U_{k, M}=\sum_{|m-M| \leq \zeta \sqrt{ } M} X_{m} \exp \left(\frac{-c(m-M)^{2}}{M}\right)-\sum_{\left|m-M_{k}\right| \leq \zeta \sqrt{ } M_{k}} X_{m} \exp \left(\frac{-c\left(m-M_{k}\right)^{2}}{M_{k}}\right) ; \\
& U_{k, M}^{(1)}=\sum_{M_{k}-\zeta \sqrt{ } M_{k} \leq m<M-\zeta \sqrt{ } M} X{ }_{m} \exp \left(\frac{-c\left(m-M_{k}\right)^{2}}{M_{k}}\right) \\
& U_{k, M}^{(2)}=\sum_{M-\zeta \sqrt{ } M \leq m<M_{k}} X \sum_{m}\left\{\exp \left(\frac{-c\left(m-M_{k}\right)^{2}}{M_{k}}\right)-\exp \left(\frac{-c(m-M)^{2}}{M}\right)\right\} ; \\
& U_{k, M}^{(3)}=\sum_{M_{k} \leq m<M} X_{m} \exp \left(\frac{-c\left(m-M_{k}\right)^{2}}{M_{k}}\right) \\
& U_{k, M}^{(4)}=\sum_{M_{k} \leq m<M} X_{m} \exp \left(\frac{-c(m-M)^{2}}{M}\right) \\
& U_{k, M}^{(5)}=\sum_{M \leq m<M_{k}+\zeta V_{k}} X X_{m}\left\{\exp \left(\frac{-c(m-M)^{2}}{M}\right)-\exp \left(\frac{-c\left(m-M_{k}\right)^{2}}{M_{k}}\right)\right\} ; \\
& U_{k, M}^{(6)}=\sum_{M_{k}+\zeta \sqrt{ } M_{k} \leq m \leq M+\zeta \sqrt{ } M} X_{m} \exp \left(\frac{-c(m-M)^{2}}{M}\right) .
\end{aligned}
$$

We note that if $k$ is sufficiently large and $M_{k}<M \leq M_{k+1}$, then $M_{k}-\zeta \sqrt{ } M_{k}$ $<M-\zeta \sqrt{ } M<M_{k}<M<M_{k}+\zeta \sqrt{ } M_{k}<M+\zeta \sqrt{ } M$, and so $\left|U_{k, M}\right| \leq \sum_{i=1}^{6}\left|U_{k, M}^{(i)}\right|$. 
First consider $U_{k, M}^{(1)}$. Setting $t=\left(8 \delta^{-2} \log k\right)^{1 / 2}$, we have from (15) that $P\left[U_{k, M}^{(1)} \geq \delta M_{k}^{1 / 4}\left(\log M_{k}\right)^{1 / 2}\right]$

$$
\begin{aligned}
& \leq\left\{\exp \left(-t \delta\left(\log M_{k}\right)^{1 / 2}\right)\right\} E \exp \left\{t M_{k}^{-1 / 4} U_{k, M}^{(1)}\right\} \\
& \leq k^{-4} \exp \left\{t^{2} M_{k}^{-1 / 2} \sum_{M_{k}-\zeta \sqrt{ } M_{k} \leq m<M-\zeta \sqrt{ } M} \sigma_{m}^{2} \exp \left(\frac{-2 c\left(m-M_{k}\right)^{2}}{M_{k}}\right)\right\} \\
& =O\left(k^{-4}\right) .
\end{aligned}
$$

Replacing $X_{m}$ by $-X_{m}$ in the above argument, we then obtain from (24) that

$$
P\left[\max _{M_{k}<M \leq M_{k+1}}\left|U_{k, M}^{(1)}\right| \geq \delta M_{k}^{1 / 4}\left(\log M_{k}\right)^{1 / 2}\right]=O\left(k^{-4}\left(M_{k+1}-M_{k}\right)\right)=O\left(k^{-3}\right) .
$$

Therefore by the Borel-Cantelli lemma, we have for $i=1$,

$$
\limsup _{k \rightarrow \infty} M_{k}^{-1 / 4}\left(\log M_{k}\right)^{-1 / 2} \max _{M_{k}<M \leq M_{k+1}}\left|U_{k, M}^{(i)}\right| \leq \delta \quad \text { a.e. }
$$

By a similar argument, we can show that (25) also holds for $i=2,3,4,5,6$. Hence (23) holds, and the desired conclusion follows from (18), (22) and (23).

Proof of Theorem 3. We first assume (12) and show that (13) and (14) both hold. Set $b_{m}(\lambda)=e^{-\lambda} \lambda^{m} /(m !)$, let $1 / 2<\alpha<2 / 3$ and let $k$ be an integer such that $k(1-\alpha)>1$. Given $\delta>0$, we choose $\epsilon>0$ such that $\epsilon k<\delta$. From (12), it follows that

$$
\lim _{n \rightarrow \infty} n^{-1 / 4}(\log n)^{-1 / 2} X_{n}=0 \text { a.e. }
$$

To prove (13), we set $M=[\lambda], h=m-M$. Since there exists $\nu>0$ such that $\Sigma_{|h|>M^{a}} b_{m}(\lambda)=O\left(\exp \left(-\lambda^{\nu}\right)\right)($ cf. [5, p. 200]), it follows from (26) that

$$
\lim _{\lambda \rightarrow \infty} \lambda^{1 / 4}(\log \lambda)^{-1 / 2} \sum_{|h|>M^{a}} b_{m}(\lambda)\left|X_{m}\right|=0 \text { a.e. }
$$

Furthermore we note that (cf. [5, p. 200])

$$
\sum_{|h| \leq M^{a}}\left|(2 \pi M)^{-1 / 2} \exp \left(\frac{-h^{2}}{2 M}\right)-b_{m}(\lambda)\right|
$$

$$
=\sum_{|h| \leq M}\left\{(2 \pi M)^{-1 / 2} \exp \left(\frac{-b^{2}}{2 M}\right)\right\}\left\{O\left(\frac{(1+|h|)}{\lambda}\right)+O\left(\frac{|b|^{3}}{\lambda^{2}}\right)\right\}=O\left(\lambda^{-1 / 2}\right) .
$$


Hence it follows from (26) that

$$
\lim _{\lambda \rightarrow \infty} \lambda^{1 / 4}(\log \lambda)^{-1 / 2}\left\{\sum_{|h| \leq M^{a}}\left|(2 \pi M)^{-1 / 2} \exp \left(\frac{-h^{2}}{2 M}\right)-b_{m}(\lambda)\right|\left|X_{m}\right|\right\}=0 \text { a.e. }
$$

Define

$$
\left.X_{n}^{\prime}=X_{n} I\left|X_{n}\right| \geq \epsilon n^{1 / 4}(\log n)^{1 / 2}\right], \quad X_{n}^{\prime \prime}=X_{n}^{I}\left[\left|X_{n}\right| \leq n^{1 / 4} /(\log n)\right]
$$

and $X_{n}^{\prime \prime \prime}=X_{n}-X_{n}^{\prime}-X_{n}^{\prime \prime}$. Let $Y_{n}=X_{n}^{\prime \prime}-E X_{n}^{\prime \prime}$. Then $E Y_{n}=0, E Y_{n}^{2}=\sigma_{n}^{2} \rightarrow$ $\sigma^{2},\left|Y_{n}\right| \leq 2 n^{1 / 4} /(\log n)=o\left(n^{1 / 4}(\log n)^{-1 / 2}\right)$ and therefore satisfies the hypothesis of the lemma (cf. [8, p. 255]). Hence

$$
\limsup _{\lambda \rightarrow \infty}(\pi \lambda)^{-1 / 4}(\log \lambda)^{-1 / 2} \sum_{|h| \leq M^{\alpha}} Y_{m} \exp \left(\frac{-h^{2}}{2 M}\right)=\sigma \text { a.e. }
$$

Noting that $E X_{1}=0$, we have for $|h| \leq M^{\alpha}$,

$$
\left|E X_{m}^{\prime \prime}\right|=\left|E X_{1} I_{\left[\left|X_{1}\right|>m^{1 / 4} /(\log m)\right]}\right| \leq m^{-1 / 2}(\log m)^{2} E\left|X_{1}\right|^{3} .
$$

Hence

$$
\lim _{\lambda \rightarrow \infty} \lambda^{1 / 4}(\log \lambda)^{-1 / 2} \sum_{|h| \leq M^{a}}\left(M^{-1 / 2} \exp \left(\frac{-h^{2}}{2 M}\right)\right)\left|E X_{m}^{\prime \prime}\right|=0 .
$$

Also by (26), $P\left[X_{n}^{\prime} \neq 0\right.$ i.o. $]=0$. Since $m^{1 / 4} /(\log m)<\left|X_{m}^{\prime \prime \prime}\right|<\epsilon m^{1 / 4}(\log m)^{1 / 2}$, we have for all large $M$,

$$
\begin{aligned}
P\left[\sum_{|h| \leq M^{a}}\left|X_{m}^{\prime \prime \prime}\right| \geq \delta M^{1 / 4}(\log M)^{1 / 2}\right] \leq P\left[\sum_{|h| \leq M} \frac{\left|X_{m}^{\prime \prime \prime}\right|}{\left(\epsilon m^{1 / 4}(\log m)^{1 / 2}\right)} \geq k\right] \\
\quad \leq P\left[X_{m}^{\prime \prime \prime} \neq 0 \text { for at least } k \text { of the indices } M-M^{\alpha} \leq m \leq M+M^{\alpha}\right] \\
\leq\left(\begin{array}{c}
1+2\left[M^{\alpha}\right] \\
k
\end{array}\right) P^{k}\left[\left|X_{1}\right|^{4}>\left(M-M^{\alpha}\right)\left\{\log \left(M-M^{\alpha}\right)\right\}^{-4}\right] \\
\quad=O\left(M^{\alpha k-k}(\log M)^{6 k}\right), \text { by the Markov inequality. }
\end{aligned}
$$

Since $k-a k>1$, an application of the Borel-Cantelli lemma then gives

$$
\limsup _{\lambda \rightarrow \infty} \lambda^{1 / 4}(\log \lambda)^{-1 / 2} \sum_{|h| \leq M^{a}} M^{-1 / 2}\left|X_{m}^{\prime \prime \prime}\right| \leq \delta \quad \text { a.e. }
$$


Hence we have proved (13). To prove (14), setting $M=[(n+1) /(q+1)]$, $c=(q+1) / 2 q$, we can use a similar argument as before (cf. [5, p. 201]).

We now prove that (13) implies (12). From (13), it is clear that $X_{n} \rightarrow$ $O(B)$ a.e. By the equivalence of (9) and (11), we have $E X_{1}=0$ and $E X_{1}^{2}$ $<\infty$. For $n=3,4, \ldots$, let $d_{m}(n)=(\pi n)^{1 / 4}(\log n)^{-1 / 2} b_{m}(n), Y_{n}=\sum_{m=1}^{n} d_{m}(n) X_{m}$, $Z_{n}=\Sigma_{m=n+1}^{\infty} d_{m}(n) X_{m}$. Since $E X_{1}=0$ and $E X_{1}^{2}<\infty$, it follows from Tchebychev's inequality that $Z_{n} \stackrel{P}{\longrightarrow} 0$. Now $Z_{n}$ is independent of $\left(Y_{1}, \ldots, Y_{n}\right)$ and $\lim \sup _{n \infty}\left|Y_{n}+Z_{n}\right|=\sigma / \sqrt{ } 2$ a.e. Hence by Lemma 1 of [7], $\lim \sup _{n \rightarrow \infty}\left|Y_{n}\right| \leq \sigma / \sqrt{ } 2$ a.e. But $Y_{n}=\sum_{m=1}^{n-1} d_{m}(n) X_{m}+d_{n}(n) X_{n}$ and $d_{n}(n) X{ }_{n} \stackrel{P}{\longrightarrow} 0$. Therefore applying Lemma 1 of [7] again, we have

$$
\underset{n \rightarrow \infty}{\lim \sup }\left|\sum_{m=1}^{n-1} d_{m}(n) X\right| \leq \frac{\sigma}{\sqrt{ } 2} \quad \text { a.e. }
$$

and

$$
\limsup _{n \rightarrow \infty}\left|d_{n}(n) X_{n}\right| \leq \sqrt{ } 2 \sigma \text { a.e. }
$$

Hence

$$
\limsup _{n \rightarrow \infty} n^{-1 / 4}(\log n)^{-1 / 2}\left|X_{n}\right|<\infty \quad \text { a.e., }
$$

and since this $\lim$ sup can only be $\infty$ a.e. of 0 a.e., it follows that $n^{-1 / 4}(\log n)^{-1 / 2} X_{n} \rightarrow 0$ a.e. Therefore $E X_{1}^{4}\left(\log ^{+}\left|X_{1}\right|+1\right)^{-2}<\infty$. It is now obvious from (13) that $E X_{1}^{2}=\sigma^{2}$. In a similar way, we can prove that (14) implies (12).

Acknowledgment. I wish to thank Professor Y.S. Chow for his valuable suggestions and encouragement.

\section{REFERENCES}

1. Y. S. Chow, Delayed sums and Borel summability of independent, identically distributed random variables, Bull. Inst. Math. Acad. Sinica 1 (1973), 207-220.

2. Y. S. Chow and T. L. Lai, Limiting behavior of weighted sums of independent random variables, Ann. Prob. 1 (1973), 810-824.

3. W. Feller, An extension of the law of the iterated logarithm to variables without variance, J. Math. Mech. 18 (1968-69), 343-355. MR 38 \#1721.

4. V. F. Gapoškin, The law of the iterated logarithm for Cesàro's and Abel's methods of summation, Teor. Verojatnost. i Primenen. 10 (1965), 449-459 = Theor. Probability Appl. 10 (1965), 411-420. MR 33 \#3336.

5. G. H. Hardy, Divergent series, Clarendon Press, Oxford, 1949. MR 11, 25.

6. P. Hartman and A. Wintner, On the law of the iterated logarithm, Amer. J. Math. 63 (1941), 169-176. MR 2, 228. 
7. T. L. Lai, Limit theorems for delayed sums, Ann. Prob. 2 (1974) (to appear).

8. M. Loève, Probability theory. Foundations. Random sequences, 3rd ed., Van Nostrand, Princeton, N. J., 1963. MR 34 \#3596.

9. V. Strassen, An invariance principle for the law of the iterated logarithm, Z. Wahrscheinlichkeitstheorie und Verw. Gebiete 3 (1964), 211-226. MR 30 \#5379.

10. R. J. Tomkins, A generalization of Kolmogorov's law of the iterated logarithm, Proc. Amer. Math. Soc. 32 (1972), 268-274. MR 45 \#1229.

DEPARTMENT OF MATHEMATICAL STATISTICS, COLUMBIA UNIVERSITY, NEW YORK, NEW YORK 10027 